\title{
Response to: "A somatization comorbidity phenotype impacts response to therapy in rheumatoid arthritis: post hoc results from the certolizumab pegol phase 4 PREDICT trial"
}

\author{
Annelise Madison ${ }^{1,2^{*}}$ (D), Barbara L. Andersen ${ }^{2}$ and Ali Ajam ${ }^{3}$
}

Curtis et al. report a post hoc analysis of data from the certolizumab pegol phase 4 clinical trial among rheumatoid arthritis patients [1], p. 4. They infer a "somatization comorbid phenotype" (SCP) among patients who, at intake, had (a) diagnoses of depression, anxiety, or neuropathic pain or (b) were receiving medication for such conditions. Notably, $43 \%$ of patients met the criteria. As the authors predict, these individuals were more likely to drop out and report adverse side effects and less likely to achieve low disease activity status. They conclude that SCP may mask the drug's true effectiveness, and thus, they question whether to "include, identify, or stratify" these patients in future clinical trials. Their operationalization of SCP and resulting conclusion are seriously flawed.

First, the authors' definition of SCP does not index somatization, or patient-reported physical symptoms with no known organic cause. Medically unexplained somatic complaints and depression/anxiety are not inherently comorbid. In this study, SCP-positive patients did not somaticize: although their clinician-rated disease activity was greater than SCP-negative individuals, they did not report greater symptom severity. Therefore, the authors' SCP grouping lacks construct validity. Disregarding the SCP label, their findings replicate prior research that links depression and anxiety with greater disease activity in RA. This study only muddied the waters by grouping dissimilar individuals and inaccurately labeling them as somaticizers.

Most troubling are the authors' three recommendations to eliminate this unwanted variance among RA patients: exclude, label, or stratify these patients. Stratification is unnecessary, as these patients comprise almost half of the population of interest and groups would be equated on this dimension if a sufficiently large sample is randomized. Identifying depressed and anxious RA patients is the only viable option they suggest-but only if the purpose is to offer supportive treatment. Excluding these patients is untenable from a biological perspective. A depressive episode increases risk for autoimmune disease onset [2] and fuels inflammation [3], while RA-related inflammation can maintain and even worsen depression [4]. An RA sample free of depression/anxiety would not represent the RA population and treatment efficacy would be artificially inflated.

There are many troubling aspects to Curtis et al.'s (2017) report. It offers no contribution to the existing literature, but instead pejoratively labels RA patients struggling with mood dysregulation and/or neuropathic pain. The final recommendations for managing these patients via research design strategies harken to a dangerous and unethical view of individual differences.

* Correspondence: annelise.madison@osumc.edu

${ }^{1}$ Institute for Behavioral Medicine Research, The Ohio State University College

of Medicine, 460 Medical Center Drive, Columbus, OH 43210, USA

${ }^{2}$ Department of Psychology, The Ohio State University, 1835 Neil Avenue,

Columbus 43210, OH, USA

Full list of author information is available at the end of the article 


\section{Authors' response}

Jeffrey R. Curtis, Christopher Herrem, Matladi N. Ndlovu, Cathy O'Brien and Yusuf Yazici

We appreciate the opportunity to respond to the misapprehensions raised by Madison et al. of our analysis. They offer critique based on a fundamental misunderstanding of both the context and intent behind our approach, which should be viewed from the perspective of the design of a rheumatoid arthritis (RA) clinical trial, not guidance for patient-centric clinical care. We share their implied assertion that the experiences of all people living with RA, including the many who also face depression and anxiety, ought to be reflected in the study of the effectiveness and safety of RA treatments.

That said, the assertion by Madison and colleagues that depression, anxiety, and medically unexplained somatic complaints that result in chronic pain "are not inherently comorbid" seems to inappropriately minimize the importance that these conditions have on the lives of RA patients. Moreover, failure to recognize them as distinct from active RA potentially limits the opportunity to address and treat them independently. Secondly, the statement that patients with the SCP phenotype did not report greater symptom severity ignores the evidence we provided in the manuscript. Baseline pain, patient global, and tender joint count were numerically higher in SCP patients. We also showed that the benefits of starting certolizumab pegol were attenuated by approximately $10-15 \%$ for the key trial outcomes (e.g., low disease activity, ACR 20/50/70). Since patient-derived assessments factor importantly in these composite RA outcomes, their statement that outcome differences were solely driven by clinician ratings seems unsupported by evidence from our study.

Additionally, Madison et al. criticized our grouping of somewhat distinct conditions into a SCP phenotype. RA trials typically desire to minimize participant burden and avoid "non-essential" data collection. Thus, information about depression, anxiety, and comorbidities associated with non-RA pain (e.g., diabetic neuropathy, spondylosis, fibromyalgia) is often lacking. For the purposes of this analysis, information classifying patients by SCP status was from the medical history and concomitant medication data collected at baseline. Prospectively collecting these data is generally not a priority in trials and, once the trial has ended, the opportunity is lost. Thus, our phenotype offers a practical approach that can be applied even retrospectively to already-collected data to identify patients who may have an attenuated response to an effective RA treatment. Indeed, we have recently shown that this concept translates to other data sources (e.g., an EHR-based registry), where concomitant fibromyalgia resulted in an attenuated treatment response following initiation of a new RA medication [5].

They find fault with our recommendation to consider the possibility of accounting for the SCP phenotype in the design of a trial. While it is true that randomization would balance $\mathrm{SCP}+$ patients between treatment groups in large samples, and thus suggest that "stratification is unnecessary," they neglect the fact that one cannot randomize patients to the SCP phenotype, and not all RA studies are randomized. Indeed, within an observational study, identifying individuals with the SCP phenotype (or its components) allows one to adjust for or otherwise control for this factor. Moreover, even with randomization, rheumatologists place high importance on not only the relative effect of RA medications versus a comparator treatment, but also on the absolute response (the "60-40-20" ACR response) [6]. Because the SCP phenotype meaningfully reduces absolute response rates by $10-15 \%$, including large numbers of patients with this phenotype in a RA therapy trial will inappropriately penalize that therapy's perceived effectiveness. Data from international studies comparing the prevalence of some comorbidities that make up the SCP phenotype (e.g., depression) have shown high variability between countries [7]. Therefore, the magnitude of the effect on RA outcomes may be substantial in clinical studies where the prevalence of SCP phenotype conditions varies widely. As for another possibility we mentioned, excluding patients with the SCP phenotype, we agree that this would excessively limit generalizability and likely would be inadvisable for most trials.

Finally, we are disappointed that Madison et al. feel that identifying mood dysregulation and/or neuropathic pain in an RA patient assigns them a "pejorative label." We respectfully disagree and would offer the perspective that rheumatologists can and should be concerned with a holistic and personalized approach to RA patient management. As part of high-quality clinical care, we believe that depression, anxiety, and non-RA-related causes of pain need to be identified and treated. However, this does not always entail changing biologic therapy nor deeming a patient's RA treatment to be ineffective. The analysis we presented quantifies the extent to which SCP-related conditions may moderate the measured effectiveness of an anti-TNF in the context of a RA clinical trial. While many RA trials are not intended to mirror clinical practice, we nevertheless strongly agree that RA patients' individual differences must be considered and valued in both research and routine practice settings. A growing number of examples exist within rheumatology where patient-centered research is at the forefront of scientific questions that are being asked and answered [8]. 


\section{Acknowledgements}

Not Applicable

\section{Funding}

Not Applicable

\section{Availability of data and materials}

Not Applicable

\section{Authors' contributions}

AM drafted the manuscript. BA was a major contributor in writing and revising the manuscript. AA reviewed and revised the manuscript. All authors read and approved the final manuscript.

Ethics approval and consent to participate

Not Applicable

\section{Consent for publication}

Not applicable

\section{Competing interests}

The authors declare that they have no competing interests.

\section{Publisher's Note}

Springer Nature remains neutral with regard to jurisdictional claims in published maps and institutional affiliations.

\section{Author details}

${ }^{1}$ Institute for Behavioral Medicine Research, The Ohio State University College of Medicine, 460 Medical Center Drive, Columbus, OH 43210, USA.

2Department of Psychology, The Ohio State University, 1835 Neil Avenue, Columbus 43210, OH, USA. ${ }^{3}$ Division of Rheumatology and Immunology, The Ohio State University College of Medicine, 480 Medicine Center Drive,

Columbus 43210, OH, USA

Published online: 20 February 2019

\section{References}

1. Curtis JR, Herrem C, Ndlovu MN, O'Brien C, Yazici Y. A somatization comorbidity phenotype impacts response to therapy in rheumatoid arthritis: post-hoc results from the certolizumab pegol phase 4 PREDICT trial. Arthritis Res Ther. 2017;19(1):215.

2. Andersson N, Gustafsson L, Okkels N, et al. Depression and the risk of autoimmune disease: a nationally representative, prospective longitudinal study. Psychol Med. 2015;45(16):3559-69.

3. Copeland WE, Shanahan L, Worthman C, Angold A, Costello EJ. Cumulative depression episodes predict later C-reactive protein levels: a prospective analysis. Biol Psychiatry. 2012;71(1):15-21.

4. Valkanova V, Ebmeier KP, Allan CL. CRP, IL-6 and depression: a systematic review and meta-analysis of longitudinal studies. J Affect Disord. 2013; 150(3):736-44.

5. Yun HCL, Xie F, Patel H, Boytsov N, Zhang X, Curtis JR. Do Patients with Moderate or High Disease Activity Escalate RA Therapy According to Treat-to-Target Principles? Results from the ACR's RISE Registry. Chicago: ACR; 2018.

6. Breedveld FC, Kalden JR. Appropriate and effective management of rheumatoid arthritis. Ann Rheum Dis. 2004;63(6):627-33.

7. Dougados M, Soubrier M, Antunez A, Balint P, Balsa A, Buch MH, Casado G, Detert J, El-Zorkany B, Emery P, et al. Prevalence of comorbidities in rheumatoid arthritis and evaluation of their monitoring: results of an international, cross-sectional study (COMORA). Ann Rheum Dis. 2014;73(1):62-8.

8. Arthritis Power available at: https://arthritispower.creakyjoints.org/. Accessed 31 Jan 2019 